\title{
DISTRIBUTIVE SUBLATTICES OF A LATTICE
}

\author{
M. KOLIBIAR
}

\begin{abstract}
There are given necessary and sufficient conditions for a subset of a lattice $L$ (or of a complete lattice $L$ ) to generate a distributive sublattice of $L$ (or a completely distributive closed sublattice of $L$ ).
\end{abstract}

1. Introduction. In the paper [4] by B. Jónsson, conditions for a subset of a modular lattice to generate a distributive sublattice are given. In the present paper there are given necessary and sufficient conditions for a subset of a lattice $L$ (or of a complete lattice $L$ ) to generate a distributive sublattice of $L$ (or a completely distributive closed sublattice of $L$ ). As a corollary we get the result of G. N. Raney [6] and H. F. J. Lowig [5].

2. Notations. (Cf. [5].) Given a function $f, R_{f}$ will denote the range of $f$. If $J$ is a set, $\left(a_{i} \mid i \in J\right)$ denotes a function on $J$ with values $a_{i}$. Its range will be denoted by $\left\{a_{i} \mid i \in J\right\}$. If $F$ is a mapping from a set $J$ onto a set of sets, $\prod F$ denotes the cartesian product of $F$, i.e. the set of all functions $i \mapsto f(i) \quad(i \in J)$, where $f(i) \in F(i)$. The sign $\prod_{i \in J} A_{i}$ means the same as $\prod\left(A_{i} \mid i \in J\right)$. If $S$ is a set of sets, $\prod S$ denotes the set of all functions $t$ on $S$ with values $t(X) \in X$ for every element $X$ of $S$. Hence

$$
\prod S=\prod(X \mid X \in S)=\prod_{X \in S} X .
$$

If $X$ is a subset of a complete lattice $L, \wedge X$ and $\bigvee X$ are, respectively, the meet and join of the elements of $X$. Given elements $a_{i}(i \in J)$ of $L$, $\bigwedge_{i \in J} a_{i}$ and $\bigvee_{i \in J} a_{i}$ mean $\bigwedge\left\{a_{i} \mid i \in J\right\}$ and $\bigvee\left\{a_{i} \mid i \in J\right\}$, respectively.

3. A complete lattice $L$ is said to be completely distributive if for every function $\left(A_{i} \mid i \in J\right), A_{i} \subset L$,

$$
\begin{aligned}
& \bigwedge \bigvee A_{i}=\bigvee\left\{\bigwedge_{i \in J} f(i) \mid f \in \prod_{j \in J} A_{j}\right\} \\
& \bigvee_{i \in J} \bigwedge A_{i}=\bigwedge\left\{\bigvee_{i \in J} f(i) \mid f \in \prod_{j \in J} A_{j}\right\}
\end{aligned}
$$

Received by the editors October 20, 1971.

AMS 1970 subject classifications. Primary 06A35, 06A23.

Key words and phrases. Lattices, complete lattices, distributive lattices, completely distributive lattices, sublattices.

(c) American Mathematical Society 1972 
These identities are equivalent with the relations

$$
\begin{aligned}
& \bigwedge_{i \in J} \bigvee A_{i} \leqq \bigvee\left\{\bigwedge_{i \in J} f(i) \mid f \in \prod_{j \in J} A_{j}\right\}, \\
& \bigvee \wedge A_{i} \geqq \wedge\left\{\bigvee_{i \in J} f(i) \mid f \in \prod_{j \in J} A_{j}\right\},
\end{aligned}
$$

since the converse relations are obvious.

REMARK 1. Let $S$ be a subset of a complete lattice. The following conditions are equivalent.

(a) (1) holds for every family $\left(A_{i} \mid i \in J\right)$ of subsets of $S$.

(b) For each set $M$ of subsets of $S$

$$
\bigwedge_{X \in M} \bigvee X=\bigvee_{t \in \Pi M} \bigwedge_{X \in M} t(X)
$$

An analogous assertation holds for the identity (2).

Proof. Obviously (a) implies (b). Suppose (b) holds. Let $J_{0}$ be such a subset of $J$ that the functions $\left(A_{i} \mid i \in J\right)$ and $\left(A_{i} \mid i \in J_{0}\right)$ have the same range and the second function is a monomorphism. Then, by (b),

$$
\bigwedge_{i \in J_{0}} \bigvee A_{i}=\bigvee\left\{\bigwedge_{i \in J_{0}} f(i) \mid f \in \prod_{j \in J_{0}} A_{j}\right\}
$$

Since to each function $f \in \prod_{j \in J_{0}} A_{j}$ a function $g \in \prod_{j \in J} A_{j}$ exists such that $\bigwedge_{i \in J_{0}} f(i)=\bigwedge_{\in J} g(i)$ we get

$$
\bigvee\left\{\bigwedge_{i \in J_{0}} f(i) \mid f \in \prod_{j \in J_{0}} A_{j}\right\} \leqq \bigvee\left\{\bigwedge_{i \in J} f(i) \mid f \in \prod_{j \in J} A_{j}\right\}
$$

Combining this with the obvious relation $\bigwedge_{i \in J} \bigvee A_{i}=\bigwedge_{i \in J_{0}} \bigvee A_{i}$ we get $\left(1^{\prime}\right)$, hence (1).

A lattice $L$ is distributive if one of the identities (1), (2) holds for finite $J$ and $A_{i}$.

LEMMA 1. Let $H=\prod\left(A_{j} \mid j \in K\right)$ and let $\varphi: H \rightarrow K$ be a mapping. Then $\in \varphi(H)$ exists such that $\{h(j) \mid h \in H, \varphi(h)=j\}=A_{j}$.

Proof. If the conclusion is false then $B_{j}=A_{j}-\{h(j) \mid h \in H$, $\varphi(h)=j\} \neq \varnothing$ for each $j \in K$. By the Axiom of Choice, $h_{0} \in H$ exists such that $h_{0}(j) \in B_{j}$ for every $j \in K$. For $j_{0}=\varphi\left(h_{0}\right)$ we get $h_{0}\left(j_{0}\right) \notin B_{j_{0}}$, a contradiction.

In [5] and [6] it is proved that one of the identities (1), (2) implies the other. By a similar argument as in [5] a stronger result can be proved:

THEOREM 1. Let $S$ be a subset of a complete lattice L. Then (1) holds 
for each function $\left(A_{i} \mid i \in J\right), A_{i} \subset S$, if and only if for each such function (2) holds.

Proof. We shall show that (1) implies (2). Consider the elements

and

$$
\begin{aligned}
& a=\bigvee_{i \in J} \bigwedge A_{i}, \\
& b=\bigwedge\left\{\bigvee f(i) \mid f \in \prod_{i \in J} A_{j}\right\}=\bigwedge\left\{\bigvee R_{f} \mid f \in \prod_{j \in J} A_{j}\right\}
\end{aligned}
$$

$$
c=\bigvee_{t \in \mathbf{n} N} \bigwedge_{X \in N} t(X)
$$

where $N=\left\{R_{f} \mid f \in \prod_{j \in J} A_{j}\right\}$. By assumption, $b=c$. Hence it suffices to show $a \geqq c$. This follows immediately from the following assertion.

(3) To every $t \in \prod N$ there exists $i \in J$ such that $A_{i} \subset R_{t}$.

This assertion follows immediately from the Lemma 1 , if we define to a given $t$ the mapping $\varphi_{t}$ of the set $H=\prod\left(A_{j} \mid j \in J\right)$ into $J$ as follows. Given $h \in H, \varphi_{t}(h)$ is an index $j$ such that $t(h)=h(j)$.

This proves the theorem.

COROLlaRY 1 ([5], [6]). In a complete lattice the identities (1) and (2) are equivalent.

By the same argument as in Theorem 1 we can prove

THEOREM 1a. Let $S$ be a subset of a lattice L. Then the identity (1) holds for every finite family $\left(A_{i} \mid i \in J\right)$ of finite subsets $A_{i}$ of $S$ if and only if for these families (2) holds.

LEMMA 2. Let $S$ be a subset of a complete lattice $L$ such that (1) holds for each family $\left(A_{i} \mid i \in J\right)$ of subsets of $S$. Let $T_{i}(i=1,2)$ be subsets of $L$ such that each element of $T_{1}\left(T_{2}\right)$ is a meet (join) of some elements of $S$. Then the identity (1) holds for each family $\left(A_{i} \mid i \in J\right)$ of subsets of $T_{1}$ and $T_{2}$ too. $A$ similar assertion holds for the identity (2).

Proof. In view of Theorem 1 and the duality it suffices to show that $\left(1^{\prime}\right)$ holds for the elements of $T_{1}$. It will be convenient to express $\left(1^{\prime}\right)$ in the form (we set $A_{i}=\left\{a_{i, j} \mid j \in K_{i}\right\}$ )

$$
\bigwedge_{i \in J} \bigvee_{j \in K_{i}} a_{i, j} \leqq \bigvee \bigwedge_{\varphi \in \Phi} \bigwedge_{i \in J} a_{i, \varphi(i)}
$$

where $a_{i, j} \in T_{1}$ and $\Phi=\prod_{i \in J} K_{i}$. Since for each $a_{i, j} \in T_{1}, a_{i, j}=$ $\bigwedge_{\alpha \in C_{i, j}} b_{\alpha}^{i, j}$, where $b_{\alpha}^{i, j} \in S$, we have to show

$$
a=\bigwedge \bigvee_{i \in J} \bigvee_{j \in K_{i}} \bigwedge b_{\alpha \in C_{i, j}}^{i, j} \leqq \bigvee \bigwedge_{\varphi \in \Phi} \bigwedge_{i \in J} \bigwedge_{\alpha \in C_{i, \varphi(i)}} b_{\alpha}^{i, \varphi(i)}=b
$$


By the assumption and Theorem 1,

$$
\bigvee_{j \in K_{i}} \bigwedge_{\alpha \in C_{i, j}} b_{\alpha}^{i, j}=\bigwedge_{h \in H_{i}} \bigvee_{j \in K_{i}} b_{h(j)}^{i, j}
$$

where $H_{i}=\prod_{j \in K_{i}} C_{i, j}$. Using (1) we get

$$
\begin{aligned}
a & =\bigwedge_{i \in J} \bigwedge_{h \in H_{i}} \bigvee_{j \in K_{i}} b_{h(j)}^{i, j} \\
& =\bigwedge_{(i, h) \in \cup_{i \in J}\left(\{i\} \times H_{i}\right)} \bigvee_{j \in K_{i}} b_{h(j)}^{i, j} \\
& =\bigvee_{\psi \in \Psi} \bigwedge_{(i, h) \in \bigcup_{i \in J}^{\left(\{i\} \times H_{i}\right)}} b_{h(\psi(i, h))}^{i, \psi(i, h)} \\
& =\bigvee_{\psi \in \Psi} \bigwedge_{i \in J} \bigwedge_{h \in H_{i}} b_{h(\psi(i, h))}^{i, \psi(i, h)},
\end{aligned}
$$

where $\Psi$ is the set of all functions $\psi$ on $\bigcup_{i \in J}\left(\{i\} \times H_{i}\right)$ such that $\psi(i, h) \in K_{i}$ for each $h \in H_{i}$. To prove $a \leqq b$ it suffices to show:

Let $\psi \in \Psi$. There exists $\varphi \in \Phi$ such that

$$
\bigwedge_{h \in H_{i}} b_{h(\psi(i, h))}^{i, \psi(i, h)} \leqq \bigwedge_{\alpha \in C_{i, \varphi(i)}} b_{\alpha}^{i, \varphi(i)} \text { for each } i \in J
$$

Pick $i \in J$ out. We apply Lemma 1 to the set $H_{i}=\prod\left(C_{i, j} \mid j \in K_{i}\right)$ and to the mapping $\varphi^{\prime}: H_{i} \rightarrow K_{i}$ defined by $\varphi^{\prime}(h)=\psi(i, h)$. Then there is $j_{0} \in \varphi^{\prime}\left(H_{i}\right)$ such that $C_{i, j_{0}}=\left\{h\left(j_{0}\right) \mid h \in H_{i}, \psi(i, h)=j_{0}\right\}$. Set $\varphi(i)=j_{0}$. If we define $\varphi(i)$ in such a way for every $i \in J$ then each element $b_{\alpha}^{i, j}$ occurring on the right side of (4) (for a fixed $i$ ) occurs on the left side, hence (4) holds.

THEOREM 2. Let $S$ be a subset of a complete lattice $A$ and let $L^{*}(S)$ be a closed sublattice (cf. [1]) of A generated by $S . L^{*}(S)$ is completely distributive if and only if for every set $M$ of subsets of $S$ one of the following identities holds.

$$
\begin{aligned}
& \bigwedge_{X \in M} \bigvee X=\bigvee_{t \in \Pi M} \bigwedge_{X \in M} t(X), \\
& \bigvee_{X \in M} \wedge X=\bigwedge_{t \in \Pi M} \bigvee_{X \in M} t(X)
\end{aligned}
$$

Proof. Obviously the condition is necessary. Suppose $S$ satisfies (5). Then (1) holds in $S$ (see Remark 1). Let $T_{1}$ be the set of all meets of subsets of $S$ and $T_{2}$ the set of all joins of subsets of $T_{1}$. Then $T_{2}$ is closed under arbitrary joins. According to Lemma $2, T_{2}$ is closed under arbitrary meets. Hence $T_{2}=L^{*}(S)$. Applying Lemma 2 successively to $T_{1}$ and $T_{2}$ we conclude that $T_{1}$ and $T_{2}$ satisfy (1) as well as (2) (by Theorem 1).

By a similar argument we get 
THEOREM 2a. Let $S$ be a subset of a lattice $A$ and let $L(S)$ be the sublattice of $A$ generated by $S . L(S)$ is distributive if and only if one of the laws (5) and (6) holds for finite sets $M$ of finite subsets of $S$.

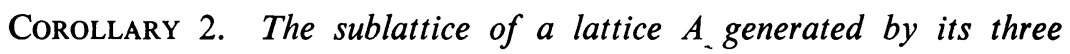
elements $x, y$ and $z$ is distributive if and only if the median equality

$$
(x \wedge y) \vee(y \wedge z) \vee(z \wedge x)=(x \vee y) \wedge(y \vee z) \wedge(z \vee x)
$$

and the six equalities arising from the equalities

$$
\begin{aligned}
& x \wedge(y \vee z)=(x \wedge y) \vee(x \wedge z), \\
& x \vee(y \wedge z)=(x \vee y) \wedge(x \vee z)
\end{aligned}
$$

by permutations, are satisfied. ${ }^{1}$

Proof. One can easily check that the equality (5) for the elements of the set $\{x, y, z\}$ reduces to those given in the Corollary 2 .

REMARK 2. None of the seven equalities in the Corollary 2 can be omitted as the examples of lattices in Figures 1 and 2 show. (The first example was communicated to the author by J. Korec.) In these examples all considered equalities hold except (7) in the first case and (8) in the second. The independence of the other equalities follows by symmetry and duality.

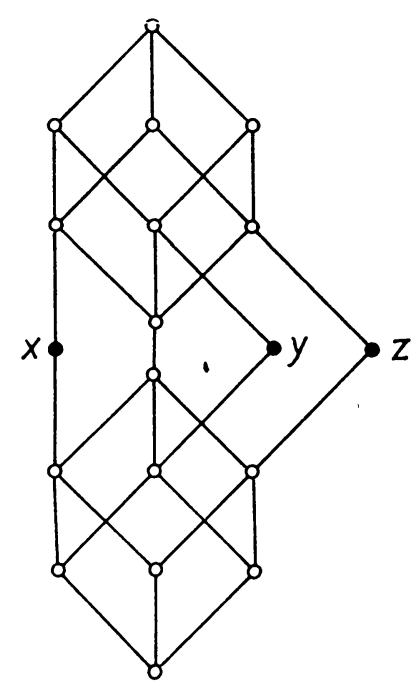

FIGURE 1

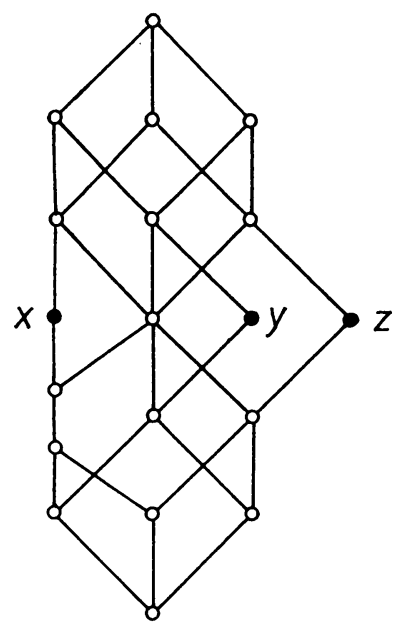

FIGURE 2

\footnotetext{
1 The proof of Theorem II.12 [1] seems to be based on the conjecture that in Corollary 2 the equality (7) can be omitted.
} 
Proposition. To any natural number $n \geqq 3$ there exists a lattice $L$ and an n-element subset $S \subset L$ which does not generate a distributive sublattice of $L$ but any proper subset of $S$ generates a distributive sublattice of $L$.

Proof (SEe [2, ReMARK 1.2]). Let $M$ be the set of all $n$-tuples $a=$ $\left(a_{1}, a_{2}, \cdots, a_{n}\right)(n \geqq 3)$ of integers such that $a_{1}+a_{2}+\cdots+a_{n}$ is even. Let $\Theta_{i}(1 \leqq i \leqq n)$ be the equivalence relations on $M$ defined as follows. $a \Theta_{i} b$ if and only if $a_{i}=b_{i}$. The set $S=\left\{\Theta_{1}, \cdots, \Theta_{n}\right\}$ does not generate a distributive sublattice of the lattice of all equivalence relations, since $\Theta_{1} \vee\left(\Theta_{2} \wedge \cdots \wedge \Theta_{n}\right)=\left(\Theta_{1} \vee \Theta_{2}\right) \wedge\left(\Theta_{1} \vee \Theta_{3}\right) \wedge \cdots \wedge\left(\Theta_{1} \vee \Theta_{n}\right)$ does not hold: If $a=(0,0, \cdots, 0), b=(1,1,0, \cdots, 0)$ then $a\left(\Theta_{1} \vee \Theta_{i}\right) b$ for each $1<i \leqq n$ but $a\left[\Theta_{1} \vee\left(\Theta_{2} \wedge \cdots \wedge \Theta_{n}\right)\right] b$ does not hold. But any subset $T$ of $S$ with $n-1$ elements generates a distributive sublattice: It can be readily seen that the set $T$ is absolutely permutable, hence it generates a Boolean sublattice (see [2, Definition 1.5 and Theorem 4.2]).

ADDED IN PROOF. Corollary 2 has been proved by O. Ore, Vierteljschr. Naturforsch. Ges. Zürich 85 (1940), p. 4. The question of independence of the conditions in Corollary 2 has not been considered by O. Ore.

\section{REFERENCES}

1. G. Birkhoff, Lattice theory, 3rd ed., Amer. Math. Soc. Colloq. Publ., vol. 25, Amer. Math. Soc., Providence, R.I., 1967. MR 37 \#2638.

2. H. Draškovičová, Permutability, distributivity of equivalence relations and direct products, Mat. Časopis Sloven. Akad. Vied 23 (1973) (to appear).

3. S. S. Holland, Jr., A Radon-Nikodym theorem in dimension lattices, Trans. Amer. Math. Soc. 108 (1963), 66-87. MR 27 \#1392.

4. B. Jónsson, Distributive sublattices of a modular attice, Proc. Amer. Math. Soc. 6 (1955), 682-688. MR 17, 341.

5. H. F. J. Lowig, Note on the self-duality of the unrestricted distributive law in complete lattices, Israel J. Math. 2 (1964), 170-172. MR 31 \#2176.

6. G. N. Raney, Completely distributive complete lattices, Proc. Amer. Math. Soc. 3 (1952), 677-680. MR 14, 612.

Department of Mathematics, Univerzita Komenského Bratislava, Bratislava, Czechoslovakia 